\title{
The Different Culture Models and Technical of Rhodeus Sinensis
}

\author{
Xiao Jiang Chen, Quan Wang* \\ Jiangsu Agri-Animal Husbandry Vocational College, Taizhou City, Jiangsu Province, China.
}

\begin{abstract}
This paper discusses three kinds of culture mode including the outdoor cement pond, indoor recirculating aquaculture and aquarium breeding, and culture related techniques are summarized here. Rhodeus sinensis, it has a very large potential for development in China. Maintaining the water quality conditions and nutritional feed is the key.
\end{abstract}

Keywords: Rhodeus sinensis; aquaculture; culture models

\section{Introduction}

Rhodeus sinensis is one of famous native fishes in China. In 1960s, it was introduced into Europe as aquarium fish, which was known as China rainbow [1]. The color is gorgeous, the shape is elegant, and it can be cultivated for ornamental fish. It was widely distributed in major river systems in China, especially rich in the Yangtze River [2]. With the impact of environmental pollution factors, their numbers have dropped sharply. Researches on Rhodeus sinensis were focused on biological habit [3,4,5,6,7] and molecular biology [8,9,10]; artificial breeding has been carried out [11].

Based on the project of Jiangsu Provincial Bureau of ocean and fishery, this paper introduces different kinds of culture models and technical as follows.

Culture conditions and treatment periods.

\section{Materials And Methods}

This study was carried out in Jiangsu Agri-animal Husbandry Vocational College. The parents of Rhodeus sinensis was selected from Lixiahe region (Fig.1). water temperature $14^{\circ} \mathrm{C}-25^{\circ} \mathrm{C}$, the dissolved oxygen concentration of 6.0-8.0 mg* $\mathrm{L}^{-1}, \mathrm{pH}$ level of 7.5-8.5.

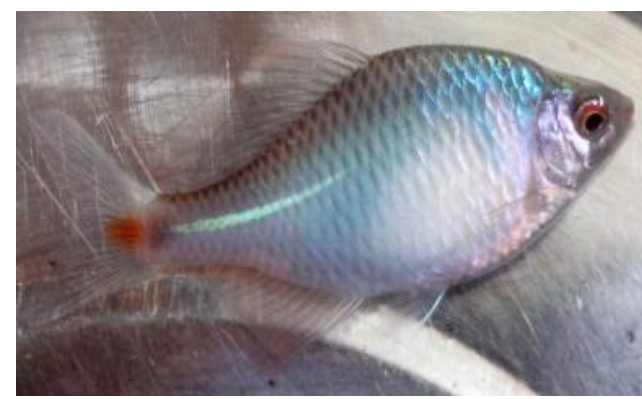

Fig.1 The parents of Rhodeus sinensis (ふ̋,body length 5-6cm)

Experimental diet.

In this experiment, artificial formulated feed was prepared with $20 \%$ of fish meal, $17 \%$ of dry tenebrio molitor, $13 \%$ of flour, $5 \%$ of pumpkin powder, $20 \%$ of corn flour, $10 \%$ of wheat bran, $2 \%$ of kelp powder, $0.45 \%$ of Calendula officinalis, $0.5 \%$ of brewer's yeast, $1 \%$ of calcium biphosphate, $0.5 \%$ of betaine, $0.5 \%$ of spirulina powder, $0.5 \%$ of red chili powder, $0.5 \%$ of garlic powder, $4 \%$ of red worms powder, $2 \%$ of fish oil, $3 \%$ of soybean oil, and $0.05 \%$ of fungicide.

\section{Outdoor Cement Pool Culture}

III. Results

\subsection{Cement pool conditions}

Cement pool is rectangular, east-west; it has 3.5 meters long, width of 1.5 meters, and height of 2 meters. The center of the cement pool is low and its surrounding is high, the sewage leakage was set in the center of the pool. The drain is wrapped around by the net to prevent the fish from slipping away. The cement pool should be equipped with microporous aeration, or the pump with oxygen stone. It can be placed 2-4 air stone per square meter, the number of stones placed in the pool depends on the density of the culture. Generally, there are natural algae attached to the cement pool, mainly for the green algae or diatoms, it can avoid the scratching surface of the fish in the process of their feeding or swimming. 


\subsection{The management of the cement pool}

In the process of breeding, due to the residual waste accumulation, bait density can lead to problems such as water quality deterioration; Harmful algal will become dominant species and harm the fish. To this, we mainly adopt the method of changing water to water diversion. The key to replace water is mainly to observe the water quality. When the color is changing from yellow to dark green or black, it is about to change water in time. From March to May, the experiment has not yet begun spawning, it usually change the water once a week. The Rhodeus sinensis spawning begins from June to August; we must focuses on the water transferring. The temperature is high at this time, and the water is rich in organics, the algae propagation goes fast, and the harmful algae appear in large numbers, such as algae and microcystin algae blooms. Under the serious condition, the water can appear like green paint material. It is composed of microcystin algae. But at the moment when the Rhodeus sinensis are spawning and mating, the frequent replacement of water is bad, so we usually add beneficial bacteria to decompose toxic and harmful substances.

\subsection{Feeding}

Outdoor cement pond aquaculture is rich in plankton. The Rhodeus sinensis can eat a lot of protozoan such as branch Angle. The fishes were fed regularly at 9:00 am and 5:00 pm daily according to their weight [12]. In reproductive season, we can also add artificial feed. The adding amount is mainly depends on the activity of the Rhodeus sinensis. When there are only few or no fish floating on the surface being feeding, we can stop feeding2. Indoor Circulating Water Aquaculture

\subsection{Indoor water recirculation system}

The circulating water filtration system is divided into four groups, each group has three barrels (Fig.2).

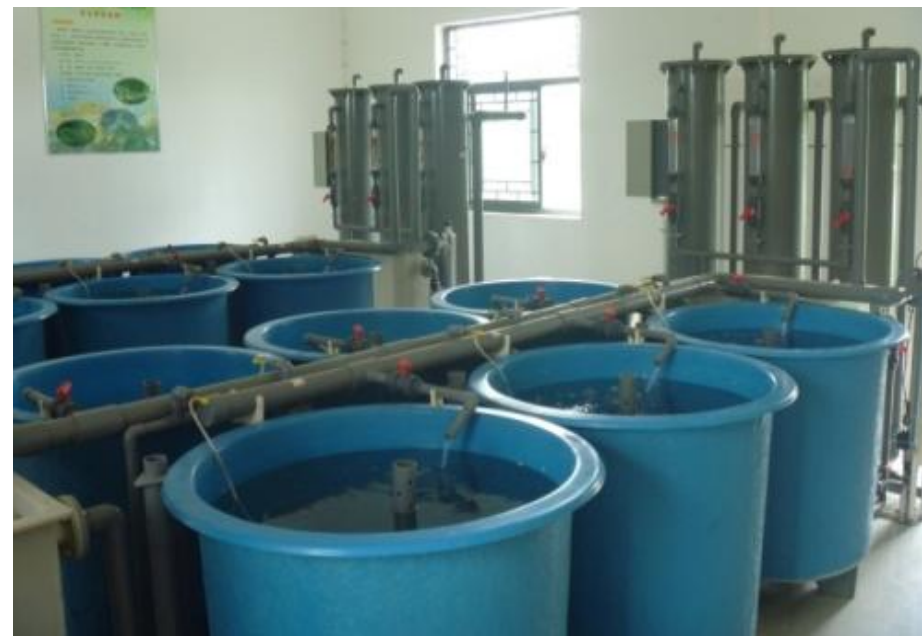

Fig.2 Indoor water recirculation system

There is a cylindrical column with holes in the middle of each barrel. It is used for sewage. The dirty water in bucket will be discharged through the hole to go. This dirty water first through the preliminary filtering unit and then flows into the biological filter, the preliminary filtering device is composed of three layers of cloth and a large number of fine sands. It is used to filter large amounts of substances in dirty water. The biological filter is to purify water by microbial decomposition, through two coarse and fine filters. Clean water will flow into the bucket from the top of the bucket, so as to form a cycle. In addition, each bucket is equipped with microporous aeration equipment to ensure adequate dissolved oxygen.

\subsection{Circulating water aquaculture management}

The daily management of the circulatory system is mainly cleaning filter cloth and material. When cleaning of the filter cloth, we'd better take out the three pieces of cloth, to wash away the dirt with the help of tap water momentum, and the effect is much better than the hand rub, and it takes a short time too. It should not be cleaned with cleaning agent. In addition, before the cleaning of the filter cloth, you can put off the circulating device. And then kick the back brake; the external water will come out from the bottom of the primary filter to get the dirt out of the sand. And the dirt will flow out of the hole, and we have to stir from time to time to avoid some of the sediment deposited on the sand, causing that it can't flow out of the hole and make it clean. In addition, the circulation device does not need to be always opened; we can add artificial intelligence control. For example, after the fish is eaten, the bait deposits, fish body secretion increased, and when we open it at this time, it can not only achieve the function of the filter cycle, but also save energy. 


\section{Aquarium Aquaculture 3.1 Aquarium}

With the development of science and technology, aquarium integrates advanced equipment, such as automatic temperature control, automatic feeding and other functions, and there are round, square, hexagonal, mini type, household type, etc, in order to meet the diversified needs from customers. In the experimental base, we used the rectangular artificial temperature aquarium, with the capacity of $0.6 * 0.3 * 0.45 \mathrm{~m}^{3}$. The principle of aquarium and circulating water filtration system is the same, which is a cycle. The difference between two is circulating system has a biological filter, but aquarium is just to use cloth filter. In addition, the oxygenation of aquarium is through a small blower which gets oxygen into box. Because the filtration and oxygenation equipment of aquarium are worse than that of circulating system, so the density of aquarium aquaculture is low, generally used for raising ornamental fish, and experimental observation.

\subsection{Aquarium management}

Daily care of the aquarium, include clean the glass cylinder and cloth. Method and points of cleaning cloth are the same as circulating water filtration system. Sewage pipe on filter device should be cleaned in time before the drain hole plugged by dirty. For the cleaning of the glass cylinder, there is a special cleaning device which has two pieces of magnet, one is inside the cylinder, the other is outside, the outside one controls the inside one to clean membrane on the glass through the gravity between two magnets. Furthermore, the change of water can be completed by siphon. Change the water has two steps: suction bottom and pumping, and these two steps cannot be reversed, because when the water pressure is not enough, it is not easy to suck residual bait feces from the bottom, unless add water which is so time-consuming. In addition, pump out half of the water and then adds into new water, but this did not change water color much, however, pump out $2 / 3$ of the water will help. It is necessary to pay attention to temperature difference when change the water, try to reduce the difference of water temperature, otherwise the stress response of fish will be great. By contrast, we found that a layer of sand at the bottom is very useful for regulating water quality. Water color has been significantly improved, and fish mortality declined. Without sediment, fish activities will roll up the bait feces, which can consume oxygen in water and breed harmful bacteria, easily lead to illness or death of Rhodeus sinensis. In addition, feeding on the side where filter water out from circulation device, because in this side, the water flow will spread the food in various places, avoiding fishes scratching surface which leading to inflammation disease when snatching. If feed on the other side, it is likely that the feed will be sucked up and filtered by the circulating system as bait feces. Furthermore, do not feed on the glass plate top of the goldfish bowl which used for shelving lamp and circulating device, because the feed will mildew after a long time and cause disease if accidentally fall and eat by fish.

\subsection{Feeding}

The daily feeding for aquarium mainly are feed, the caliber size of Rhodeus sinensis is $2-3 \mathrm{~cm}$, so the particle size of the feed should be appropriate. In addition, algae can also be fed. The ratio of algae to water is 1 : 5 to $1: 10$, and $5 \mathrm{~g} / \mathrm{m}^{3}$ compound fertilizer also should be added with 24 hours of light source. Put several air stones into the cylinder which not for the purpose of increasing oxygen, but for making $\mathrm{CO}_{2}$ better into the water and the sinking algae floating out to get light.

\section{Environmental conditions of water}

\section{Conclusion}

Suitable aquaculture environments for Rhodeus sinensis, water temperature from $20^{\circ} \mathrm{C}$ to $30^{\circ} \mathrm{C}$, dissolved oxygen above $5 \mathrm{mg} / \mathrm{L}$, $\mathrm{pH} 7.8$ to 8.4 , and transparency from $40 \mathrm{~cm}$ to $60 \mathrm{~cm}$, ammonia nitrogen under $200 \mu \mathrm{g} / \mathrm{L}[10]$. Detect physical and chemical indicators regularly. Use microbiological preparations every two weeks. Microbial preparation is added 50 times water, then flow to the bottom of the pool with a pipe, used for disease prevention and improvement of water environment.

\section{Nutrient feed}

According to analyze component of wild Rhodeus sinensis wastes, we found there were chitin shells which belong to crustacean, but almost no Spirulina. Chlorella prototype showed that the Rhodeus sinensis can effectively digest Spirulina and Chlorella. In the feeding process we found Rhodeus sinensis have a characteristic of eating carrion, such as rotting corpse from mussels and fish, and the growth was very fast after feeding, which showed that although the Rhodeus sinensis are omnivorous fish but they have highly requirements for feed protein. The nutrition demand of Rhodeus sinensis, including $32 \%$ crude protein, $10 \%$ crude fat, $42 \%$ carbohydrate, $10 \%$ water [4].

Ornamental Rhodeus sinensis have special requirements for feed. Firstly, it needs rich nutrition to maintain fish body color. Secondly, Rhodeus sinensis seasonal leave eggs in clams for breeding needs, so 
prolong breeding time can effectively maintain the populations. Thirdly, it can enhance immunity and disease resistance for fish [13]. Adding Spirulina and Chlorella in feed, can significantly improve fish's immunity and capacity of disease resistance. After feeding with Spirulina and Chlorella, the fish body color did not change obvious throughout the year, and the length of breeding from the original eight months extended to ten months.

The Rhodeus sinensis have smaller mouth, shorter head, and smaller size. In the production process of feed particle, the diameter is about $0.5 \mathrm{~mm}$; the length is about $1.5 \mathrm{~mm}$. The appropriate hardness of the feed is it can keep in water for at least 3 minutes after dispersing [14].

\section{The prevention and treatment of common diseases}

There are two aspects of the main cause of diseases of the Rhodeus sinensis. Firstly, the deterioration of water quality; secondly, caused by trauma. The common diseases mainly are water mildew. Mainly occurred in the late winter and early spring and the water temperature suitable for breeding is $13-18^{\circ} \mathrm{C}$, mainly due to the fish body surface injury, coupled with the deterioration of water quality and then harmful substances in the water will further harm the fish body. In the early stage of the fish which contracted the disease will be restless, and the body will secrete mucus too. But this time the thing does not appear which looks like the white cotton that could be observed by naked eyes. With the continuous absorption of nutrients from the fish body, the hyphae grow constantly. Finally, the fish body was covered with a gray outer mycelium, the sick fish will swim around the walls of pond madly, with the loss of appetite, consuming too much, and dying with exhaustion. Control method: when capturing, transporting the Rhodeus sinensis, we should try to avoid mechanical damage, do the full pool splash with a mixture of baking soda and salt.

The outdoor cement pool is appropriate for large-scale cultivation of Rhodeus sinensis, ample sunshine can promote the growth of natural feed, and the physique, body color of Rhodeus sinensis are better than that of fish indoor. Indoor circulating water aquaculture is a kind of intensive, efficient mode, using modern biological technology to provide an expected good environment for aquaculture species. The key to the success of these three culture models is to maintain its good water quality and nutrition feed.

\section{Acknowledgment}

The authors are thankful to the Jiangsu Agri-animal Husbandry Vocational College for providing infrastructural facility and financial support (Grant NO: NSFYB1407). It is grateful to Ocean and Fishery Bureau of Jiangsu Province for financial support (Grant NO: 00000215010).

\section{References}

[1]. Wang, Q., \& Chen, X. (2015). Biological characteristics and propagative technologies of rhodeus sinensis. Advance Journal of Food Science \& Technology, 7(9), 668-675.

[2]. Wang, J., Wang, Q., Feng, Q., Huang, A., Zhu, G., \& Xiong, L., et al. (2012). Acute toxicity of glutaraldehyde and benzalkonium bromide on rhodeus sinensis. Guizhou Agricultural Sciences.

[3]. Guan, T. L and B. A. Afzelius. 1991. The spermatozoon of the Chinese bitterling, Rhodeus sericeus sinensis (Cyprinidae, Teleostei). J. Submicrosc. Cytol. Pathol. 23: 351-356.

[4]. Shen, J. Z. 2000. Experimental Investigations in to Some Reproduction Aspects of Chinese Bitterling, Rhodeus sinensi. J. Huazhong Agri. Univ. 19: 494-496.

[5]. Zhang, T and Z. Li. 2002. Age, growth, and reproduction of the bitterling (Paracheilognathus imberbis) in a shallow Chinese lake. J. Freshwater. Ecol. 17: 501-505.

[6]. Zeng, Y., H. Z. Liu and J. Z. Shen. 2006. Choice of oviposition sites on the gills of mussels by bitterling. Curr. Zool. 52: 272-27

[7]. Pateman-Jones, C., M. B. Rasotto, M. Reichard, M. Reichard, C. Liao, H. Liu, G. ZIĘBA and C. Smith. 2011. Variation in male reproductive traits among three bitterling fishes (Acheilognathinae: Cyprinidae) in relation to the mating system. Biol. J. Lin. Soc. 103: $622-632$.

[8]. Ueda, T., N. Mashiko and H. Takizawa. 1997. Ag-NOR variation in chromosomes of Chinese bitterlings, Rhodeus lighti and Tanakia himantegus (Cypriniformes, Cyprinidae). Ichthyol. Res. 44: 302-305.

[9]. Saitoh, K., T. Ueda and R. Arai. 2000. Highly repetitive elements from Chinese bitterlings (genus Rhodeus, Cyprinidae). Genes. Genet. Syst. 75: 349-355.

[10]. Yang, X., Z. Ma and L. Xie. 2013. Complete mitochondrial genome of the Chinese bitterling Rhodeus sinensis (Cypriniformes: Cyprinidae). Mitochondrial DNA, 26(4), 647-648.

[11]. Akai, Y and R. Arai. 1998. Rhodeus sinensis, a senior synonym of R. lighti and R. uyekii (Acheilognathinae, Cyprinidae). Ichthyol. Res. 45: 105-110..

[12]. Hussain, S. M., M. Javed, S. Asghar, M. Hussain, S. Abdullah, S. A. Raza and A. Javid. 2010. Studies on growth performance of metals mixture stressed Cirrhina mrigala in earthen ponds. Pak. J. Agri. Sci. 47: 263-270.

[13]. Peng, J., Cao, Z., \& Shijian, F. U. (2014). The locomotive lateralization and its stability of rhodeus sinensis günther. Journal of Chongqing Normal University, 31(04).

[14]. Chen, X. J., Zhu, Y. G., \& Zhao, Z. M. (2013). The influence of micropore oxygen aeration on the pond water quality environment.Advance Journal of Food Science \& Technology,5(11). 